\title{
Genetic Profiling in Heterogeneous High-Grade Adenoid Cystic Carcinoma Identifies NOTCH1 Inhibition as a Therapeutic Strategy
}

\section{Yu Wang}

Shanghai Jiao Tong University School of Medicine

\section{Bao Sun}

Shanghai Jiao Tong University School of Medicine

Chunye Zhang

Shanghai Jiao Tong University School of Medicine

\section{Ronghui Xia}

Shanghai Jiao Tong University School of Medicine

\section{Lizhen Wang}

Shanghai Jiao Tong University School of Medicine

\section{Jingjing Sun}

Shanghai Jiao Tong University School of Medicine

\section{Ting Gu}

Shanghai Jiao Tong University School of Medicine

\section{Zhen Tian}

Shanghai Jiao Tong University School of Medicine

Jiang Li ( $\square$ lijiang182000@126.com )

Shanghai Jiao Tong University https://orcid.org/0000-0002-5539-8760

\section{Research}

Keywords: Adenoid Cystic Carcinoma, Heterogeneity, Solid type, High-Grade Transformation, PDXs

Posted Date: November 13th, 2020

DOl: https://doi.org/10.21203/rs.3.rs-104544/v1

License: (c) (i) This work is licensed under a Creative Commons Attribution 4.0 International License. Read Full License 


\section{Abstract \\ Background}

High-grade adenoid cystic carcinomas (ACCs), consisting mostly of the solid type along with the occasional high-grade transformation (ACC-HGT) type, are highly aggressive and heterogeneous tumors. Because of the rarity of the tumors, therapeutic strategy guided by genetic profiles based on next generation sequencing (NGS) has been not published on these tumors.

\section{Results}

Forty-four tissues microdissected from 36 high-grade ACC cases (31 solid type ACCs and 5 ACC-HGTs) were included in our study. The solid components from the 31 solid ACCs, were enriched for mutations of genes in the NOTCH (NOTCH1, 68\%; SPEN 23\%) and the chromatin-remodeling pathway and were absent of the myoepithelial cell differentiation. The cases with NOTCH1 mutation exhibited strong NCID expression, which was associated with poor distant metastasis-free survival. BRCA2 mutation was observed in $23 \%$ of solid ACCs, conferring sensitivity to PARP inhibitors. From 5 of the solid ACCs, the intratumor heterogeneity was delineated between the cribriform/tubular and the solid components. NOTCH1 and FGFR2 mutations as well as NOTCH2 amplification were restricted to the solid component indicating the clonal selection within the same tumor. From 2 aggressive solid ACCs the subclones evolved in progression for local relapse and distant metastasis, although they manifested close genomic resemblance to primary tumors. Five tumors of ACC-HGTs were characterized by a loss of biphasic ductal-myoepithelial differentiation in HGT area and enriched for alterations in the NOTCH, P53 and PI3K pathways. Guided by the genetic profiles, the preclinical efficiency of a gamma-secretase inhibitor BMS906024 was evaluated in the ACC patient-derived xenografts (PDXs) models with activating NOTCH1 mutation and it demonstrated robust antitumor effects.

\section{Conclusion}

This is the first report to describe the intratumor heterogeneity within a solid ACC as well as the intertumor evolution of clones among the primary/recurrent/metastatic tumors. Different from conventional ACCs, high-grade ACCs should be approached with a distinct therapeutic strategy particularly targeting NOTCH1 by gamma-secretase inhibitors. Microdissecting the highest grade component guided by histology is a highly recommended tumor sampling strategy and facilitates the detection of key molecular targets.

\section{Background}

Adenoid cystic carcinoma (ACC) is the most common malignancy of the salivary gland, accounting for $30.42 \%$ of all salivary malignant neoplasms in the Chinese population ${ }^{1}$. Histological studies established that ACC is a heterogeneous tumor. Variations may be observed in the cell types, the growth pattern, the 
numbers of mitoses, and the distribution of necrosis and stromal hyalinization. Heterogeneity exists not only among tumors, but also within a tumor. The patterns of inter- and intratumor heterogeneity determine different biological and diagnostic effects in the course of treatment and clinical outcomes. Therefore, identifying and validating key genetic targets to assess intra- and inter tumor genomic heterogeneity is critical for prognostication and therapeutic stratification of ACC patients.

ACC is characterized by a dual population of cells: basaloid cells with myoepithelial/basal cell differentiation and luminal/epithelial cells ${ }^{2}$. Based on the histological morphology, there are 3 main growth patterns: cribriform, tubular, and solid, which often coexist in different proportions ${ }^{3}$. A subgroup of ACCs - about $20 \%$ of all ACCs- is termed a solid type or high-grade ACC if more than $30 \%$ of the tumor consists of a solid component ${ }^{4,5,6}$. This solid type of ACC is prone to poor prognosis, advanced stage, and distant metastasis 7,8 . Another much rarer and aggressive distinct entity of high-grade ACC with lesions of transformation into poorly differentiated adenocarcinoma or undifferentiated carcinoma has been described using the designation "ACC with high-grade transformation (ACC-HGT)" over the past decade ${ }^{9,10}$. It is histologically characterized by pleomorphism, prominent necrosis and mitotic rate even higher than that of solid patterned $A C C^{11}$. The majority of high-grade ACC patients experience progressive disease with no effective therapy available ${ }^{10,12,13}$. Due to the rarity of the solid type and high-grade transformation subtype of ACC (high-grade ACC in this study), information on key molecular events of high-grade ACC is scarce. Concerted efforts to determine molecular targets for effective treatment are being mounted.

Whole exome sequencing (WES) of ACC samples has shed light on the genetic landscape of this disease. Overall, $A C C$ is a quiet tumor with a low exonic somatic mutation rate and wide mutational diversity ${ }^{14}$. To date, the recurrent MYB/MYBL1-NFIB translocation, leading to a highly expressed myb protein, is the main genomic hallmark of ACC $(28-59 \%)^{15-18}$. Recently, alterations in the NOTCH signaling pathways, particularly NOTCH1 mutations (10\%-26.3\%) in recurrent and/or metastatic ACC have been revealed ${ }^{18-20}$. However, no genetic profile data based on next generation sequencing (NGS) analysis have been published on high-grade salivary ACCs. Also, the intra- (different components within a tumor) and intertumor (primary/recurrent/metastatic tumor in the same patient) heterogeneity of these genetic alterations has not been evaluated.

To achieve this goal and to the best of our knowledge, we investigated the largest sample size (44 microdissected samples from 36 patients, 31 solid type ACCs and 5 ACCs-HGT) of the high-grade salivary ACCs using the NGS detection and focused on the molecular alterations potential for targeting therapy. Additionally, for the first time in known publication history, we reported the genetic heterogeneity between different growth patterns within the same solid ACC tumors, and characterized the consistency and evolution of genetic alterations among the primary/recurrent/metastatic tumors. Guided by the genetic features of the high grade ACC patients, the preclinical efficiency of a gamma-secretase inhibitor BMS906024 was evaluated in the PDXs models. Our study highlighted the importance of taking intra- and 
intertumor heterogeneity of high-grade ACCs into consideration for both diagnostic and therapeutic purposes.

\section{Results}

\section{The clinical characteristics of 31 solid type ACC cases}

The clinical pathologic characteristics and follow-up information of 31 solid type ACC patients (solid type component $>30 \%$ ) are presented in Table 2. The mean age at presentation was 56.1 years (range: 31 to 89 years) with a male predominance (male to female ratio of 2.4:1). The solid type ACC cohort consisted of 26 tumors that were predominantly solid pattern (more than 80\%) and 5 tumors (ACC4809, 4802, 4810, 4813 and 4806) that comprised a roughly equal mixture of cribriform/tubular and solid histological forms. These tumors, prone to developing at the maxilla $(13 / 31,42 \%)$, were usually grossly aggressive with extraglandular extension from maxilla sites and with bone involvement at sinonasal or palatine sites. The maximum tumor diameter ranged from 1.2 to $8 \mathrm{~cm}$ with a median size of $4 \mathrm{~cm}$. Neck dissections were performed in addition to surgical excision in 3 cases when imaging tests indicated lymph node metastases. Distant metastases were clinically noted in sixteen patients, though only 1 case with lung metastases was biopsy proven. Focal necrosis was present in 20 cases (20/31, 64.5\%). 16 tumors presented perineural invasion $(16 / 31,51.6 \%)$ and 9 tumors had vascular invasion $(9 / 31,29.0 \%)$. The leading cause of death in these cases was local relapse with skull base infiltration.

\section{The genetic profiles of solid type ACC}

To further delineate the molecular profile of solid type ACC, a comprehensive molecular analysis of solid components microdissected from 31 solid type ACCs was performed. A total of 316 genomic alterations (307 mutations + 9 copy number alterations) were identified in the solid components of 31 tumors with an average of 10.2 per tumor. A total of 110 genomic alterations (103 mutations +7 copy number alterations) with potentially actional significance were included, in which 30 of the 31 tumors $(96.8 \%)$ harbored at least one with an average of 3.7.

Several genes were identified in which the alterations were significantly enriched in the solid type ACCs (Figure 1A) and lollipop plots of key mutated genes were shown in Figure 1C. NOTCH1, a critical regulator of cell fate determination and various developmental processes, was the most frequently mutated gene in $21 / 31(67.7 \%)$ cases. Forty somatic mutations in NOTCH1 were found including 16 frame-shifts, 6 inframe insertions/deletions, 14 missense mutations and 4 nonsense mutations. 14 samples (13/21, 61.9\%) displayed multi-hit somatic mutations to the NOTCH1 gene, of which 13 samples were predicted to be NOTCH1 activating, in keeping with its potential oncogenetic role. Thirty-one of these NOTCH1 genetic alterations occurred in the extracellular domain, heterodimerization domain (HD) and PEST domain that have been identified in multiple cancer types including T-cell acute lymphoblastic leukemia, leading to gamma-secretase inhibitor (GSI) sensitivity. This finding suggests that they were gain-offunction mutations. The four nonsense mutations were all predicted to generate premature stop codons that could result in truncation mutations and disrupt the PEST domain, causing a modest increase in 
activity. Another NOTCH1 alteration in Patient 1959 that disrupted ligand binding led to the loss of the ankyrin repeats, which are necessary for $\mathrm{NOTCH} 1$ function and predicted to be inactivating. The four other NOTCH1 mutations in the EGF-like extracellular domain of the NOTCH receptor have not been wellcharacterized, and their effect on function is unclear, although similar alterations have been reported in the context of cancer, which may indicate biological relevance. Furthermore, another member of the NOTCH pathway, SPEN, which was found to repress the transcriptional activity of NOTCH as a tumor suppressor, was mutated in $7 / 31(23 \%)$ cases. Other members of the NOTCH family with a lower prevalence of alteration were also observed: NOTCH3 (4 cases, mutation, 11.1\%) and NOTCH2 (1 mutation, $2.8 \%)$. Generally, approximately $77.4 \%$ (24/31) of cases harbored an alteration in the broader NOTCH pathway, including FBXW7. Mutations in genes important in histone modification and chromatinremodeling were also significantly enriched in solid type ACC, including CREBBP (11 cases, 35\%), EP300 (9 cases, 29\%), KDM6A (8 cases, 26\%) and MLL2 (6 cases, 19\%). BRCA2 mutations, which confer sensitivity to PARP inhibitors, were detected in $23 \%(7 / 31)$ of solid type ACCs, but were quite uncommon in the cribriform/tubular type.

Moreover, ten copy number alterations (CNAs) were detected, including losses involving $5 q$ and $18 q$ and gains involving $1 p, 8 q$ and $10 q$. On chromosome 1p, the locus of the NOTCH2 gene (1p12), the HSD3B1 (1p12) and the MYCL1 gene (1p34.2) showed an amplification in 6\% (two cases) of the solid type ACCs. Notably, two patients with NOTCH2 amplification were not only likely to develop lung metastasis, but also had a far higher likelihood of developing metastasis in the liver. Another region of interest on chromosome 8q is the locus of the MYC and RAD21 genes (8q24), which showed an amplification in 6\% (two cases) of solid type ACCs. Region 5q11.2, where the MAP3K1 gene is located, showed a loss in 1 case. The chromosome 18 where contained the SMAD4 gene was deleted in 1 case as well (Supplementary Figure 2).

We then focused on the status of fusion genes identified in solid type ACCs. MYB-NFIB fusion was detected in 6 cases $(6 / 31,19 \%)$ of the solid type and demonstrated that they all involved MYB exon 14 and NFIB exon 9. In Patient 1955, rearrangements of MYB with APOO were found. Additionally, MYB truncation was identified in Patient 4819. Collectively, MYB rearrangement may not be the key genetic alteration in solid type ACC, although substantial complexity of the structural rearrangements in the MYB gene was indicated.

\section{Heterogeneity of molecular alterations in the distinct components within solid type ACCs}

Intratumor heterogeneity, leading to variable treatment responses, has been one of the major challenges in treating cancer. Comprehensive analysis of multiregional samples aids in understanding intratumoral heterogeneity. To this end, the consistency and discrimination of genetic alterations between the cribriform/tubular component and solid components microdissected separately within a high-grade tumor were assessed in 5 cases (ACC4809, 4802, 4810, 4813 and 4806) (Figure 2A).

Phylogenetic tree analysis demonstrate that all 5 ACCs had some genetic alterations that were common among other histological components but with an enrichment in the cancer cell fractions from the 
cribriform component to the solid component and other alterations that were component-specific. In ACC 4813, two frame-shift mutations in NOTCH1 (R1594fs and P1582fs) and one missense mutation in MRE11A (P629S) were restricted to solid type ACC, with cell fraction of $24 \%, 5 \%$ and $41 \%$ respectively. Mutations in $\mathrm{NOTCH} 1$ (P1582fs, 5\%) were subclonal, indicating the presence of intratumor genetic heterogeneity. In addition, the nonsense mutation in CSF3R (Q707*) became clonal in the solid type ACC, with cancer cell fractions ranging from $40 \%$ in the cribriform/tubular ACC to $90 \%$ in the solid type ACC (Figure 2B , 2C and 2D). ACC 4809 is a tumor derived from the sweat glands of skin near the nose. Three nonsense mutations in genes EP300 (Q341*), SPEN (R702*) and HSD3B1(R250*) existed in both components of ACC4809, but there was an enrichment in the cancer cell fractions from $8 \%, 14 \%$ and $74 \%$ in the cribriform component to $45 \%, 76 \%$ and $88 \%$ in the solid component respectively. A frameshift in NOTCH1 (F1736fs) was detected in the cribriform component and missed in the solid component, whereas the copy number amplification in $\mathrm{NOTCH} 2$ was acquired in the solid component (Figure $2 \mathrm{~B}, 2 \mathrm{C}$ and 2D Supplementary Figure 2). In ACC 4810, A frame-shift mutation in MLL2 (R2188fs) and a missense mutation in PPP2R2A (R252Q) were restricted to the solid component with the cancer cell fractions of $32 \%$ and $38 \%$ respectively (Figure $2 B$ and $2 C$ ). In ACC 4806 , a missense and subclonal nonsense mutation in TP53 (C275Y and R342*), was restricted to cribriform ACC. Akin to ACC 31, the missense mutation in NOTCH1 (L1709P) was acquired in the progression to solid type ACC with a cancer cell fraction of $62 \%$ (Figure 2B and 2C). In ACC 4802, 1 splice site mutation in KDM6A (splice site $564+1 G>C$ ) and 3 missense mutations in PIK3C2G (R781C), PTCH1 (D898N) and TET2 (T1114S) were found in the cribriform ACC component. In fact, the PIK3C2G (R781C), PTCH1 (D898N) and TET2 (T1114S) mutations may be the founder genetic events, as they were present in both the cribriform and the solid components with the cancer cell fraction ranging from $48 \%$ to $52 \%$, whereas the KDM6A (splice site $564+1 \mathrm{G}>\mathrm{C}$ ) mutation was restricted to only the cribriform component. However, four mutations targeting 3 genes that affect bona fide cancer genes, FGFR2 (Y375C), NOTCH1 (A2478fs, A1742fs) and CREBBP (E1000*), were verified to be restricted to solid component (Figure 2B ,2C and 2D).

In 2 of the 5 cases (ACC4813 and ACC4802), the MYB-NFIB fusion gene involving MYB exon 14 and NFIB exon 9 was detected in both the cribriform/tubular and solid components, suggesting that MYB-NFIB fusion was an early event in the tumorigenesis of primary ACC.

\section{Intertumor (primary/recurrent/metastasis) heterogeneity of solid type ACCs}

Solid type ACC is known to be related to poor prognosis, local relapse, and distant metastasis. To characterize the genetic evolution that drives its propensity for recurrence or metastasis of the solid type, two aggressive ACCs were included in our study. These two cases were both diagnosed as solid type ACC with infiltrative borders in the primary tumors. Patient 4820 (ACC 4820) and Patient 1958 (ACC 1958) showed local recurrence at 11 and 16 months after surgery respectively. Distant metastases to the lung were clinically noted in Patient 1958 at 13 months after surgery and then proven by biopsy. We performed NGS on the primary and paired recurrent tumor specimens of both patients and the distinct lung metastases of Patient 1958. 
In ACC 1958, the MYB-NFIB fusion gene and 9 somatic mutations were all detected in the primary, recurrent and metastatic tumor specimens. However, one missense mutation and one frameshift mutation in NOTCH1 (L1709P, S2467fs) were both significantly enriched in the metastatic specimen with cancer cell fractions of $45-46 \%$ in primary ACC, $45-48 \%$ in local reoccurring ACC vs. $65-68 \%$ in metastatic ACC, suggesting that NOTCH1 may be a driver gene in the progression of ACC distant metastasis. In addition, a subclonal mutation of SPEN (R1475*), a member of the NOTCH pathway as a known histone modification gene, was found in the metastatic specimen, further supporting the role of the NOTCH pathway in ACC (Figure 2D and 2E).

In ACC 4820, mutations in AR and CHEK2 were identified as the founder genetic event, being present and clonal in both the primary and recurrent specimens with cancer cell fraction of more than $90 \%$. Akin to ACC1958, NOTCH1 mutation was also detected in both the primary and recurrent specimen, although with no enrichment in the cancer cell fraction. In addition, chromosome 1, the area in which the NOTCH2 and HSD3B1 genes are located, showed amplification restricted to the local recurring specimen (Figure 2D and 2E, Supplementary Figure 2).

From the above, we concluded that primary, recurrent and metastatic specimens shared consistent main alterations. SPEN, a member associated with the NOTCH pathway, is not only important in the tumorigenesis of solid type ACC, but also plays an early and/or an initiating tumorigenic role in primary tumor development and metastatic progression. In addition, the amplification of NOTCH2 and HSD3B1 on chromosome 1 may promote the local relapse of solid type ACC.

\section{Immunohistochemical characteristics in the different components of the solid type ACCs}

To identify the pathologic features of distinct components (cribriform/tubular and solid) within a solid type ACC, an immunohistochemical study of the myoepithelial marks of p63 and p40, together with the luminal markers CK7, and Ki-67 was carried out. The solid component was diffusely positive for CK7 ( Figure $3 \mathrm{~A}, \mathrm{~d}$ and $\mathrm{f}$ ) and negative or weak for p63 (Figure 3A, $g$ and $\mathrm{i}$ ) and p40 (Figure 3A, $\mathrm{j}$ and $\mathrm{I}$ ) with the loss of the biphasic appearance, while CK7 (Figure 3A, $d$ and e) and p63/p40 (Figure 3A, j and k) highlighted the epithelial and myoepithelial cells respectively in the lesions of conventional ACC. Ki-67 immunostaining indicated that the proportion of proliferating cells was more than $10 \%$ (positive nuclear staining) in the solid component and $<5 \%$ in the cribriform/tubular component (Figure $3 \mathrm{~A}, \mathrm{~m}, \mathrm{n}$ and $\mathrm{o}$ ). The same was true of p63/p40 and CK7 immunostainings in the solid pattern of the other 26 solid type ACCs (Supplementary Figure 3). These results indicated that gland differentiation with the absence of myoepithelial cell differentiation was a major feature of solid type ACCs.

\section{FISH analysis in the different components of the solid type ACCs}

To confirm the gene rearrangement results from NGS in 31 solid type ACCs, we investigated MYB status by means of FISH. In the 7 cases that NGS showed MYB-NFIB/APOO fusion, FISH analysis displayed two kinds of patterns: one was the typical pattern with an intact signal (fused orange/green signals) and a split signal (separated orange and green signals), indicating a breakpoint within the MYB gene (4/7), and 
the other was an atypical pattern with one fusion signal and one green signal, indicating the loss of the $3^{\prime}$ end (3/7). In the case NGS presented MYB truncation (case 4819), the atypical pattern with one fusion signal and one green signal was observed (Data not shown). Intriguingly, in ACC 4813 and ACC 4802 which arranged in distinct growth patterns, the typical patterns with an intact signal and a split signal were shown in the cribriform components (Figure 3B-b and Supplementary Figure 4b), while the atypical pattern with one fusion signal and one green signal, indicating the loss of the 3 ' end (2/5), was present in the solid component (Figure 3B-a and Supplementary Figure 4a).

\section{NOTCH1 mutation and NCID expression}

The correlation between NOTCH1 mutation and prognosis was analyzed in solid type ACCs. KaplanMeier analyses showed that patients with NOTCH1/NOTCH2 alteration were inclined to have lower distant metastasis-free survival rates $(\mathrm{P}=0.032)$ than those with no $\mathrm{NOTCH} 1 / \mathrm{NOTCH} 2$ mutation, but there was no difference in the overall survival and recurrence-free survival between the patients in the two groups (Figure. 4A). The association between NOTCH1 mutations and NOTCH1 activation was then determined. The immunostaining of NCID, an established marker of NOTCH1 activation was performed. A statistically significant association between NOTCH1 mutations and NICD positivity was identified (Figure 4B). The H score of nuclear NICD1 expression in 20 tumors with NOTCH1 potentially activating mutations was much higher than that in 11 tumors with NOTCH1 wild-type or predicted NOTCH1 mutation to be inactivating ( $190.5 \pm 13.05$ vs $71.8 \pm 9.61$, P凶0.0001, Figure $4 \mathrm{C})$. There was no difference in NICD1 expression between tumors with one and multi-hit NOTCH1 mutations predicted to be activating $(165.7 \pm 16.88, N=7$ vs $203.8 \pm 17.19, N=13$, Figure 4D).

\section{The genetic profiles of ACC with high-grade transformation}

Recently, several studies reported that a distinct entity of ACC with high-grade transformation (HGT) often gradually transforms from solid type ACC, but other than p53 mutation ${ }^{21}$, the molecular genetic mechanisms responsible for these transformations remain largely unknown. Therefore, our study included 5 cases of ACC with HGT to concentrate on genetic alterations (Table 3 ). The mean age of the five patients was 47.4 years (range, 20-64 years), and all patients were male. The mean size of the tumor was $4.1 \mathrm{~cm}$ (range, 1.9-5.5 cm). In all cases, the transformed components comprised more than $30 \%$ of the entire lesion and displayed larger and more pleomorphic, vesicular nuclei. Micropapillary growth was noted and necrosis was common (Figure 5B-a, b and Figure 5C-a, b). Neck dissections were performed in addition to surgical excision in 3 cases in which imaging tests and subsequent pathological slices indicated lymph node metastasis.

The high-grade transformation component was the target lesion that was microdissected for NGS. MYBNFIB fusion existed in only one case (Figure 5A). However, the multi-hit mutations of NOTCH1 predicted to be activating were observed in 3 cases and a NOTCH1 rearrangement of unclear significance was found in another case. Since NOTCH1 mutations are fundamental and critical in the tumorigenesis and progression of solid type ACC, our results proposed that ACC with HGT was not an abrupt transition from 
low-grade ACC but may be a gradual transformation from solid type ACC. Most importantly, somatic mutations of genes including PIK3CA, PIK3R1 and PPP2R2A associated with the PI3K/AKT pathway were identified in 4 cases of ACCs with HGT. PIK3CA encodes the PI3K catalytic subunit p110a, and PIK3R1 encodes the PI3K regulatory subunit p85a, and PPP2R2A is a potent negative regulator of AKT. Mutations in PIK3CA occurred in the helical domain (E545K) and the kinase domain (H1047R) and mutations in PIK3R1 that occurred in iSH2 domains (N564D) were all hotspots for the activation of the canonical PI3K pathway. In the remaining case, p53 mutation and FGFR2 amplification played an important role in the progression to high-grade transformation. Above all, we concluded that the gain of function mutations in p53, PI3K/AKT related genes or FGFR2 amplification were essential events in the progression from solid type ACC to HGT ACC.

As described above, the expression levels including CK7, p63, NCID, the PI3 Kinase p110a, p53 and pAKT ${ }^{\mathrm{T} 308}$ were analyzed. P63 immunostaining was negative and CK7 immunostaining was weakly or moderately positive in the high-transformation grade area, indicating loss of the biphasic appearance. Ki67 immunostaining was more than $50 \%$ positive, indicating a high proliferation ability. The cases with NOTCH1 mutations showed high expression of NCID, while that with $\mathrm{NOTCH} 1$ rearrangement presented negative expression of NCID. Strong and diffuse expression of p53 was observed in Patient 4821. Of the 4 tumors with mutations in $\mathrm{PI} 3 \mathrm{~K} / \mathrm{AKT}$ related genes, positivity was observed for $\mathrm{p} 110 \mathrm{a}$ and $\mathrm{pAKT} \mathrm{T}^{\mathrm{T} 308}$ with a high intensity and extent of staining (Figure 5B and 5C).

\section{The in vivo evaluation of NOTCH1 inhibitor BMS-906024 in high grade ACC PDXs}

A panel of ACC PDXs were established by our group. PDX 34 was from ACC 4820 which harbored a multihit mutation of NOTCH1. The HE staining and IHC staining of PDX 34 was done to confirm the histological consistency to ACC 4820. The xenograft tumor exhibited solid type and diffusely positive for CK7 and negative for p63. As well, it showed a high expression of NCID indicating the activation of $\mathrm{NOTCH}$ signaling pathway. As NOTCH1 is the most frequently mutated gene in high grade ACCs, a preclinical evaluation of $\gamma$-secretase inhibitor (GSI) BMS-906024 inhibiting NOTCH signaling was conducted on PDX 34. Single-agent treatment with BMS-906024 $(8.5 \mathrm{mg} / \mathrm{kg})$ or cisplatin $(5 \mathrm{mg} / \mathrm{kg})$ were performed in the PDX models for 28 days. BMS-906024(TGI=48\%) reduced the volume and weight of xenograft tumors and exhibited a better tumor growth inhibitory effect than cisplatin (TGI=12\%). In addition, the toxicities of BMS-906024 and cisplatin were well tolerated during the treatment period. To confirm that the TGI induced by BMS-906024 was a consequence of a downregulation of NOTCH1, we performed IHC staining of NCID in the PDX tumors after the treatment. Treatment of PDX 34 with BMS906024 inhibited NCID expression much better than cisplatin and the control. However, cell proliferation, which assessed by Ki67 staining, was reduced to varying degrees in cisplatin and BMS-906024 groups.

\section{Discussion}

Increasing experimental and clinical evidence suggests that some cancers are not homogeneous in their cell composition but are composed of heterogeneous cell subtypes. The heterogeneity appears to 
contribute to the variations in its molecular aberrations, cell surface markers, growth rates, and ability to generate intra- and extracellular proteins, complicating patient prognostication and therapeutic response. ACC is a heterogeneous tumor of histologic morphology or biological characteristics. However, comprehensive analysis of the heterogeneity of this tumor has been constrained due to the lack of structured tumor sampling in space and time. In our study, we located different regions based on histologic patterns within high-grade ACCs through imaging modalities for better selection of specimens for genomic analysis and further verified the subclonal enrichment or evolution among the primary tumors, recurrent tumors and lung metastatic deposits. We also better characterized solid type and HGT ACC through a comprehensive pathological and molecular analysis and identified NOTCH inhibition as a therapeutic strategy in PDXs.

Whole exome sequencing or whole genome sequencing are common methods for analyzing molecular alterations in ACC using fresh tumor samples and corresponding blood samples as controls. However, intratumor genetic heterogeneity existes in every malignant tumor. ACC, often presents mixed tumors in which the cribriform/tubular and solid components coexist in different proportions. F1CDx is a next generation sequencing (NGS) based in vitro diagnostic device using DNA isolated from formalin-fixed paraffin embedded (FFPE) tumor tissue specimens, allowing us to find and microdissect the target area under the microscope, making our results more accurate and reliable. Our results showed that clonal shifts or potential clonal selection involved in the genetic alterations of NOTCH1, MLL2, FGFR2, PPP2R2A, CREBBP and NOTCH2 amplification may take place exclusively in the solid component, but not in the cribriform/tubular component. The immunohistochemical analysis revealed that loss of myoepithelial cell differentiation, was a major feature of the solid component compared with the cribriform/tubular component. A study by Yalcin-Ozuysal reported that NOTCH activation facilitated mammary differentiation toward the luminal epithelial fate by downregulating p63, which might explain the loss of p63 immunostaining in the progression to solid histology ${ }^{22}$. Moreover, the subclones evolve at varying temporal stages of tumor progression for both local relapse and distant metastasis, although they manifest close genomic resemblance to ancestral primary tumors. These findings, together with the considerable intratumoral and intertumoral genetic differences, underscore the importance of an reasonable sampling strategy for prognostication and clinical management.

Adenoid cystic carcinoma is deemed to arise from progenitor intercalated duct reserve cells, which are able to change into ductal luminal and myoepithelial tumor cells. We propose that intratumoral heterogeneity of solid type ACC (mixture of solid pattern and cribriform/tubular pattern) is the result of multidirectional differentiation of the progenitor intercalated duct reserve cells. The cellular composition of cribriform/tubular pattern is similar to that in the "terminal differentiation" stage of a developing salivary gland, including the myoepithelial or basaloid cells, while that of the solid pattern represents the proliferation of the less differentiated intercalated duct cells without the differentiation of myoepithelial or basaloid cells.

Another important aspect of our study is the identification of somatic mutations, copy number variants and rearrangements in solid type ACC, suggesting a distinct entity different from convention 
cribriform/tubular ACC. The recurrent MYB/MYBL1-NFIB translocation, leading to a highly expressed myb protein, is known to be the main genomic hallmark of ACC (28-59\%). However, in our cohort of solid type ACC, MYB rearrangement was present in 6/31(19\%) samples, much lower than the overall incidence, suggesting that MYB rearrangement may not be the major molecular alteration in solid type ACC. Moreover, we identified a number of mutations in cancer genes that were enriched in solid type ACC. Previous investigations revealed the landscape of salivary ACC, in which NOTCH1 mutation was uncommon, and in recurrent/metastatic ACC, approximately $26 \%$ cases harbored this alteration. In the solid type ACC of breast investigated by Julie Massé, NOTCH1 was the most frequently mutated gene, and the rate was $28 \%^{23}$. However, according to our study, salivary solid type ACC showed powerful evidence of strong selective pressure for $\mathrm{NOTCH} 1$ mutations, $68 \%$ of which harbored this mutation ( $65 \%$ activating mutation) with frequent multi-hit mutations. Another NOTCH pathway member, SPEN, was also observed to have a high mutation rate $(19 \%)$. Furthermore, a statistically significant association between NOTCH1 mutations and NICD positivity (an established marker of NOTCH1 activation) was identified. Above all, we concluded that NOTCH pathway-related gene alterations, especially NOTCH1 and SPEN mutations were the central mediators of tumorigenesis in solid type ACC.

NOTCH1 inhibitors and gamma-secretase inhibitors (GSIs) may be potential therapeutic approaches in cases of $\mathrm{NOTCH} 1$ activating mutations ${ }^{24-26}$. Complete responses to the GSI AL101 were achieved in a patient with T-cell acute lymphoblastic leukemia (T-ALL) harboring a NOTCH1 HD domain mutation ${ }^{27}$ and in a patient with gastroesophageal junction adenocarcinoma harboring multiple NOTCH1 mutations ${ }^{28}$. On the basis of clinical data in non-Hodgkin lymphoma, NOTCH1 activating alterations may be associated with sensitivity to the FDA-approved PI3K inhibitor copanlisib; this is further supported by limited preclinical data that suggest that $\mathrm{NOTCH} 1$ may be a negative regulator of PTEN ${ }^{29,30}$. A patient with tracheal ACC harboring two somatic NOTCH1 mutations achieved a partial response to the NOTCH1targeting antibody brontictuzumab; upon progression, an additional NOTCH1 mutation and an FBXW7 inactivating mutation were identified ${ }^{20}$. Hence, guided by the molecular characteristics of high-grade ACCs, the preclinical efficiency of a gamma-secretase inhibitor BMS-906024 was evaluated in the ACC patient-derived xenografts (PDXs) with activating NOTCH1 mutation. BMS-906024 exhibited a robust antitumor effects and NOTCH1 inhibition may be a potentially effective approaches in solid type ACC with a high frequency of $\mathrm{NOTCH} 1$ mutations.

Similarly, multiple genes involved in chromatin modification that have been previously reported including CREBBP, KDM6A and EP300 were consistently enriched but at a significantly higher frequency with mutations in solid type ACC. CREBBP and EP300 (structurally similar to CREBBP) encode a ubiquitously expressed transcriptional coregulatory protein that interacts with multiple transcription factors and can couple control of gene expression to chromatin remodeling via its histone acetyltransferase activity ${ }^{31}$. KDM6A encodes a histone $\mathrm{H} 3$ lysine 27 demethylase UTX, which functions as a transcriptional regulator as well ${ }^{32}$. Cooccurrence mutations between these genes involved in chromatin modification and NOTCH1 were often observed $(16 / 21,76 \%)$ and the former implied a possible biological synergistic effect that resulted in cancer progression by recruiting NOTCH1 transcriptional regulators. Notably, BRCA2

Page 11/28 
mutations were detected in $23 \%$ of solid type ACC, which was quite uncommon in the cribriform/tubular type. The BRCA2 tumor suppressor gene encodes a protein that regulates the response to DNA damage ${ }^{33}$. Inactivating mutations in BRCA2 can lead to the inability to repair DNA damage and loss of cell cycle checkpoints, which can lead to tumorigenesis ${ }^{34}$. The loss or inactivation of BRCA2, confers sensitivity to PARP inhibitors such as niraparib, which demonstrated clinical activity for patients with breast, prostate, and pancreatic cancer ${ }^{35-37}$ and may be another novel molecular target for high-grade ACCs.

Adenoid cystic carcinoma with high-grade transformation is another subgroup of ACC although it is very rare. The striking clinical features of ACC-HGT was the high propensity for lymph node metastasis present, which was in 3 of the 5 cases and with shortened survival ${ }^{38}$. Investigations of the molecular alterations underlying this extremely aggressive behavior are minimal. P53 and cyclin D1 protein expression were previously reported to the markers of ACC-HGT, but not all ACC-HGT harbored P53 or cyclin D1 alteration or protein overexpression. In our study, p53 mutation and protein overexpression were observed in one of the five cases of ACC-HGT. Alterations of NOTCH1 were observed in 4 of the 5 cases. Since NOTCH1 mutations are fundamental and critical in the tumorigenesis of solid type ACC, our results proposed that ACC with HGT was not an abrupt transition from low-grade ACC, but may be a gradual transformation from solid type ACC. Moreover, PI3K/AKT pathway-related genes including PIK3CA, which encodes the PI3K catalytic subunit $\mathrm{p} 110 \mathrm{a}$ and PIK3R1, which encodes the PI3K regulatory subunit $\mathrm{p} 85 \mathrm{a}^{39}$ observed here in 3 of the 5 cases have been characterized as activating, are predicted to be driver mutations in ACC-HGT. Therapies targeting PI3K or AKT may be effective for these cases of ACC-HGT. Another mutation in PPP2R2A, which encodes B55alpha, a B regulatory subunit of the tumor suppressor protein phosphatase 2 (PP2A) was detected in one case that cooccurred with the NOTCH1 mutation. Activation of PP2A can simultaneously inhibit the PI3K and MAPK pathways ${ }^{40}$. Therefore, combination therapy, taking the gamma-secretase inhibitors with PI3K/mTOR kinase inhibitors or PARP inhibitors, represents a promising therapeutic strategy for the treatment of ACC-HGT.

\section{Conclusion}

Our study delineated the genetic profiles of the high-grade salivary ACCs using the NGS detection and focused on the molecular alterations potential for targeting therapy. This is the first report to describe the intratumor heterogeneity focusing on solid types within an ACC as well as the intertumor evolution of dominant clones among the primary/ recurrent/ metastatic tumors. Different from conventional ACCs, high-grade ACCs should be approached with a distinct therapeutic strategy particularly targeting NOTCH, BRCA2 or PI3K signaling pathways. Microdissecting the highest grade components guided by the histological morphology is a highly recommended tumor sampling strategy and facilitates the detection of key molecular targets.

\section{Materials And Methods}

\section{Cases and tissues}


Patients diagnosed with ACC were reviewed from the archives of the Ninth People's Hospital affiliated to Shanghai Jiao Tong University during 2000-2019. A total of 36 high-grade ACC patients with available follow-up records were selected for this study, and the corresponding paraffin-embedded ACC specimens were obtained from the Department of Oral Pathology, the Ninth People's Hospital, following the approval of the University Institutional Ethics Committee according to histologic criteria ${ }^{38,41}$. All patients signed the informed consent. The cases in our study were all bona fide high-grade ACC. Thirty-one solid type ACCs included 26 tumors that were predominantly solid pattern (more than $80 \%$ ) and five tumors that comprised a roughly equal mixture of cribriform/tubular and solid histological forms whch were sufficient in both quantity and morphologic distinction to allow for microdissection as 2 separate targets. A third group consisted of 5 tumors with high-grade transformation (ACC-HGT) diagnosed according to the criteria described by Seethala et $\mathrm{al}^{13}$ displaying target areas that occupied at least $30 \%$ of the specimen. The tissue samples in our study were displayed in Table 1. Patients were all HPV negative based upon HPV detection by PCR-RDB via the Human Papillomavirus Genotyping Kit for 23 types (Yaneng Biosciences, Shenzhen, China). These patients were treated with surgical resection followed by postoperative radiation. All diagnostic blocks and slides of ACCs were centrally reviewed by 3 pathologists, and morphologically distinct areas of each tumor were identified.

\section{Immunohistochemistry}

Immunohistochemistry was performed using the EnvisionTM detection kit (Dako, USA) as described previously ${ }^{1}$. The details of the primary antibodies used in the study are listed in Supplementary Table 1. Three pathologists independently analyzed the data set, and a consensus in the evaluation from at least two of the three pathologists was considered acceptable. Nuclear staining of MYB, NICD1, p63, p40, p53 and Ki-67 and nuclear and cytoplasmic staining of p-AKT were assessed. CK7 and PI3 kinase p110a expression was confirmed in the cytoplasm. The percentage of stained nuclei labeled with Ki-67 and P53 was identified in three hotspot areas chosen by counting the median value at $40 \times$ magnification. The immunostaining of MYB, NICD1, CK7, S100, p-AKT and PI3 kinase p110a was graded according to the $\mathrm{H}$ score calculated by multiplying the percentage of positive tumor cells by the intensity of staining. The intensity of the staining was graded from 0 to $3(0$, negative; +1 , weak; +2 , moderate; +3 , strong). Therefore, the staining scores ranged from 0 to 300 (0, negative; $1-100$, weak; $101-200$, moderate; 201300 , high). Staining of p63 and p40 was assessed according to their presence. If absent, it was assessed as negative. If present, it was assessed by its distribution pattern as (a) diffuse (all the tumor cells were positive), (b) peripheral (the periphery but not the center was positive, Supplementary Figure 1), or (c) peripheral and central (it surrounded the tumor structures and the cavities within the tumor structures) according to the reference ${ }^{42}$.

\section{Microdissection and DNA extraction}

Tumor slides on which solid component and cribriform/tubular component could be clearly recognized within the same tumor were carefully selected. Ten-micrometer-thick sections of FFPE representative blocks of tumor tissues from each case were stained with hematoxylin. The target area of each case was 
microdissected separately with a sterile needle under a stereomicroscope (Olympus) to ensure the tumor cell content was more than $80 \%$. Genomic DNA from each tumor was extracted using the DNeasy Blood \& Tissue Kit (Qiagen, Dusseldorf, Germany), according to the manufacturer's instructions. More than $50 \mathrm{ng}$ of DNA extracted from FFPE-embedded tissue was considered qualified for the following NGS detection.

\section{Mutation-analysis pipeline}

All tissue samples were analyzed via FoundationOne ${ }^{\circledR} \mathrm{CDx}(\mathrm{F} 1 \mathrm{CDx})$, the product of Foundation Medicine. F1CDx is a next generation sequencing (NGS) in vitro diagnostic platform for the detection of substitutions, insertion, and deletion alterations (indels) and copy number alterations (CNAs) in 324 genes, and the selection of rearrangements of 34 genes, as well as genomic signatures including microsatellite instability (MSI) and tumor mutational burden (TMB) using DNA isolated from formalinfixed paraffin embedded (FFPE) tumor tissue specimens. In brief, more than $50 \mathrm{ng}$ of DNA was extracted from FFPE-embedded tissue consisting of $20 \%$ or more tumor cells. Adaptor-ligated DNA underwent hybrid capture for the entire coding region of selected cancer-related genes plus select introns from genes frequently rearranged in cancer (FoundationOne). Mutational prevalence was adjusted for the gene composition of the FoundationOne panel that was used. Captured libraries were sequenced to a median exon coverage depth of more than 500x using Illumina HiSeq platforms. Resultant sequences were analyzed for short variants (base substitutions, indels), copy number alterations (focal amplifications and homozygous deletions), and select gene rearrangements using the hg19 reference genome.

\section{Fluorescence in situ hybridization}

FISH assay for the MYB gene was performed using the DAKO Histology FISH Accessory Kit (Agilent Technologies) according to the manufacturer's instructions. Interphase molecular cytogenetic studies using a commercially available MYB Dual Color Break-Apart Probe (cat no. ZTV-Z-2143-200; ZytoVision

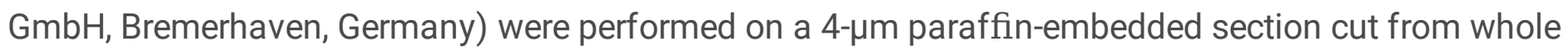
section blocks. The probe is a mixture of two directly labeled probes hybridizing to the 6q23.2-q23.3 band. The orange fluorochrome directly-labeled probe hybridizes distally, and the green fluorochrome directly-labeled probe hybridizes proximal to the MYB breakpoint cluster region. Analysis was conducted as previously described ${ }^{43}$. In an interphase nucleus lacking a translocation involving the 6q23.2-q23.3 band, two orange/green fusion signals are expected representing two normal (nonrearranged) 6q23.2q23.3 loci. A cell number $>30$ among $1{ }^{\prime} 00$ isolated cells indicated $M Y B$ abnormalities. MYB abnormalities present either with typical rearrangement (one fusion, and another with separate orange and green signals), deletion (one fusion and only one other orange or green signal) or amplification (more than two orange and/or green signals) translocation.

\section{Phylogenetic Trees}

The intra- and intertumor comparisons were carried out by the $\mathrm{R}$ statistical programming language (v3.0.2) on the confirmed somatic mutations. The distribution matrixes for the construction of the clonal phylogenetic trees were generated with $\mathrm{R}$ statistic programming based on the gene mutation counts 
among different tissues within a patient. The sorting strategy utilized that of published studies ${ }^{44}$. Upon completion of mutation sorting, phylogenetic trees were constructed for each patient to depict the ancestral relationships of mutations and the lengths of trunks and branches.

\section{Establishment of NOTCH1-activated PDXs from ACCs and NOTCH1 inhibitor efficacy evaluation in vivo}

Fresh tumor samples from ACC patients were collected and immediately immersed in cold DMEM containing $5 \%$ Penicillin and streptomycin. Tumor tissues were minced into approximately $3 \times 3 \times 3-\mathrm{mm}^{3}$ pieces and were implanted subcutaneously into the flank region of BALB/C mice (female, 4-6 weeks old). Successfully engrafted tumor models were then passaged. The passage directly harboring the patient samples was termed P0; subsequent generations were consecutively defined as P1, P2, P3.etc.

The Mice harboring the $\mathrm{P} 3$ tumor tissues were randomized into different groups when the tumors reached an average volume of 100-110 $\mathrm{mm}^{3}$ and were orally givern BMS-906024 (NOTCH1 inhibitor, $8.5 \mathrm{mg} / \mathrm{kg}$ ) (HY-15670, MedChemExpress, USA), cisplatin (5 mg/kg) (HY-17394, MedChemExpress, USA), and water (as the control). The tumor size was measured twice a week for 28 days and was calculated using the formula $T V=$ width $^{2} \times$ length $/ 2$. When the treatment was ended, tumor growth inhibition (TGI) was calculated as follows: $\mathrm{TGI}=1-[(\mathrm{TV} f$, treated $-\mathrm{TVi}$, treated $) /$

(TVf, control - TVi, control)] $\times 100 \%$, where TVf is the average tumor volume at the end of the experiment, and TVi is the average tumor volume at the start of treatment.

\section{Statistical analysis}

The statistical analyses were processed using the GraphPad Prism 6 software or SPSS 17.0 statistical software. Groups were compared using two-tailed Student's t-test. p values $<0.05$ were considered statistically significant. Overall survival, distant metastasis-free survival, and recurrence-free survival were compared with the Kaplan-Meier method.

\section{Declarations}

\section{Ethics approval and consent to participate}

ACC specimens were obtained from the Department of Oral Pathology, the Ninth People's Hospital, following the approval of Independent Ethics Committee of Shanghai Ninth People's Hospital affiliated to Shanghai Jiaotong University, and each patient signed the informed consent. The animal experiments were conducted following the ethical standards and national guidelines and were approved by the Committee on Ethics of Animal Experiments of the Ninth People's Hospital Affiliated to Shanghai Jiaotong University.

\section{Consent for publication}

All authors are in agreement with the content of the manuscript and consent to publication. 


\section{Availability of data and material}

All data generated and/or analysed during this study are included in this article and supplementary materials. The original sequencing data from Foundation Medicine are confidential as the company rule, but the related analysis data have been uploaded in the supplementary material.

\section{Competing interests}

We declare that there is no conflict of interest.

\section{Funding}

This project was funded by the National Natural Science Foundation of China under the grant nos. 81872187, the Fundamental research program funding of Ninth People's Hospital affiliated to Shanghai Jiao Tong university School of Medicine under the grant nos. JYZZ110 and the National Natural Science Foundation of China under the grant nos.81702694.

\section{Authors' contributions}

Yu Wang: Specimens collections, data analysis, preclinical PDX drug expreiment and writing

Sun Bao: PDX establilshment and analysis

Chunye Zhang, Ronghui Xia and Lizhen Wang: Histological analysis and IHC analysis

Jingjing Sun: FISH analysis

Ting Gu: IHC staining

Zhen Tian: experiment design

Jiang Li: experiment design and analysis of the data

\section{Acknowledgements}

The important contributions of our students and colleagues in this research are gratefully acknowledged and special thanks are extended to Foundation Medicine for the contributions to sequencing.

\section{References}

1. Wang Y, Zhang CY, Xia RH, Han J, Sun B, Sun SY et al. The MYB/miR-130a/NDRG2 axis modulates tumor proliferation and metastatic potential in salivary adenoid cystic carcinoma. Cell death \& disease 2018; 9(9): 917.

2. Coca-Pelaz A, Rodrigo JP, Bradley PJ, Vander Poorten V, Triantafyllou A, Hunt JL et al. Adenoid cystic carcinoma of the head and neck-An update. Oral oncology 2015; 51(7): 652-61. 
3. van Weert S, van der Waal I, Witte BI, Leemans CR, Bloemena E. Histopathological grading of adenoid cystic carcinoma of the head and neck: analysis of currently used grading systems and proposal for a simplified grading scheme. Oral oncology 2015; 51(1): 71-6.

4. Patey DH, Thackray AC. The treatment of parotid tumours in the light of a pathological study of parotidectomy material. The British journal of surgery 1958; 45(193): 477-87.

5. Perzin $\mathrm{KH}$, Gullane P, Clairmont AC. Adenoid cystic carcinomas arising in salivary glands: a correlation of histologic features and clinical course. Cancer 1978; 42(1): 265-82.

6. Atallah S, Casiraghi O, Fakhry N, Wassef M, Uro-Coste E, Espitalier F et al. A prospective multicentre REFCOR study of 470 cases of head and neck Adenoid cystic carcinoma: epidemiology and prognostic factors. European journal of cancer (Oxford, England : 1990) 2020; 130: 241-249.

7. Matsuba HM, Simpson JR, Mauney M, Thawley SE. Adenoid cystic salivary gland carcinoma: a clinicopathologic correlation. Head \& neck surgery 1986; 8(3): 200-4.

8. Nascimento AG, Amaral AL, Prado LA, Kligerman J, Silveira TR. Adenoid cystic carcinoma of salivary glands. A study of 61 cases with clinicopathologic correlation. Cancer 1986; 57(2): 312-9.

9. Seethala RR, Cieply K, Barnes EL, Dacic S. Progressive genetic alterations of adenoid cystic carcinoma with high-grade transformation. Archives of pathology \& laboratory medicine 2011; 135(1): 123-30.

10. Nagao T. "Dedifferentiation" and high-grade transformation in salivary gland carcinomas. Head and neck pathology 2013; 7 Suppl 1(Suppl 1): S37-47.

11. Hellquist H, Skálová A, Barnes L, Cardesa A, Thompson LD, Triantafyllou A et al. Cervical Lymph Node Metastasis in High-Grade Transformation of Head and Neck Adenoid Cystic Carcinoma: A Collective International Review. Advances in therapy 2016; 33(3):357-68.

12. Sato K, Ueda Y, Sakurai A, Ishikawa Y, Kaji S, Nojima T et al. Adenoid cystic carcinoma of the maxillary sinus with gradual histologic transformation to high-grade adenocarcinoma: a comparative report with dedifferentiated carcinoma. Virchows Archiv : an international journal of pathology 2006; 448(2): 204-8.

13. Seethala RR, Hunt JL, Baloch ZW, Livolsi VA, Leon Barnes E. Adenoid cystic carcinoma with highgrade transformation: a report of 11 cases and a review of the literature. The American journal of surgical pathology 2007; 31(11): 1683-94.

14. Ho AS, Kannan K, Roy DM, Morris LG, Ganly I, Katabi N et al. The mutational landscape of adenoid cystic carcinoma. Nature genetics 2013; 45(7): 791-8.

15. Togashi Y, Dobashi A, Sakata S, Sato Y, Baba S, Seto A et al. MYB and MYBL1 in adenoid cystic carcinoma: diversity in the mode of genomic rearrangement and transcripts. Modern pathology : an official journal of the United States and Canadian Academy of Pathology, Inc 2018; 31(6): 934-946.

16. Mitani Y, Liu B, Rao PH, Borra VJ, Zafereo M, Weber RS et al. Novel MYBL1 Gene Rearrangements with Recurrent MYBL1-NFIB Fusions in Salivary Adenoid Cystic Carcinomas Lacking t $(6 ; 9)$ Translocations. Clinical cancer research : an official journal of the American Association for Cancer Research 2016; 22(3): 725-33. 
17. Stephens PJ, Davies HR, Mitani Y, Van Loo P, Shlien A, Tarpey PS et al. Whole exome sequencing of adenoid cystic carcinoma. The Journal of clinical investigation 2013; 123(7): 2965-8.

18. Ho AS, Ochoa A, Jayakumaran G, Zehir A, Valero Mayor C, Tepe J et al. Genetic hallmarks of recurrent/metastatic adenoid cystic carcinoma. The Journal of clinical investigation 2019; 129(10): 4276-4289.

19. Sajed DP, Faquin WC, Carey C, Severson EA, A HA, C AJ et al. Diffuse Staining for Activated NOTCH1 Correlates With NOTCH1 Mutation Status and Is Associated With Worse Outcome in Adenoid Cystic Carcinoma. The American journal of surgical pathology 2017; 41(11): 1473-1482.

20. Ferrarotto R, Mitani Y, Diao L, Guijarro I, Wang J, Zweidler-McKay P et al. Activating NOTCH1 Mutations Define a Distinct Subgroup of Patients With Adenoid Cystic Carcinoma Who Have Poor Prognosis, Propensity to Bone and Liver Metastasis, and Potential Responsiveness to Notch1 Inhibitors. Journal of clinical oncology : official journal of the American Society of Clinical Oncology 2017; 35(3): 352-360.

21. Costa AF, Altemani A, Vékony H, Bloemena E, Fresno F, Suárez C et al. Genetic profile of adenoid cystic carcinomas (ACC) with high-grade transformation versus solid type. Cellular oncology (Dordrecht) 2011; 34(4):369-79.

22. Yalcin-Ozuysal O, Fiche M, Guitierrez M, Wagner KU, Raffoul W, Brisken C. Antagonistic roles of Notch and p63 in controlling mammary epithelial cell fates. Cell death and differentiation 2010; 17(10): 1600-12.

23. Massé J, Truntzer C, Boidot R, Khalifa E, Pérot G, Velasco V et al. Solid-type adenoid cystic carcinoma of the breast, a distinct molecular entity enriched in NOTCH and CREBBP mutations. Modern pathology : an official journal of the United States and Canadian Academy of Pathology, Inc 2019.

24. Debeb BG, Cohen EN, Boley K, Freiter EM, Li L, Robertson FM et al. Pre-clinical studies of Notch signaling inhibitor R04929097 in inflammatory breast cancer cells. Breast cancer research and treatment 2012; 134(2): 495-510.

25. Groth C, Fortini ME. Therapeutic approaches to modulating Notch signaling: current challenges and future prospects. Seminars in cell \& developmental biology 2012; 23(4): 465-72.

26. Krop I, Demuth T, Guthrie T, Wen PY, Mason WP, Chinnaiyan P et al. Phase I pharmacologic and pharmacodynamic study of the gamma secretase (Notch) inhibitor MK-0752 in adult patients with advanced solid tumors. Journal of clinical oncology : official journal of the American Society of Clinical Oncology 2012; 30(19): 2307-13.

27. Knoechel B, Bhatt A, Pan L, Pedamallu CS, Severson E, Gutierrez A et al. Complete hematologic response of early T-cell progenitor acute lymphoblastic leukemia to the $\mathrm{Y}$-secretase inhibitor BMS906024: genetic and epigenetic findings in an outlier case. Cold Spring Harbor molecular case studies 2015; 1(1): a000539.

28. Gavai AV, Quesnelle C, Norris D, Han WC, Gill P, Shan W et al. Discovery of Clinical Candidate BMS906024: A Potent Pan-Notch Inhibitor for the Treatment of Leukemia and Solid Tumors. ACS medicinal chemistry letters 2015; 6(5): 523-7. 
29. Dreyling M, Morschhauser F, Bouabdallah K, Bron D, Cunningham D, Assouline SE et al. Phase II study of copanlisib, a PI3K inhibitor, in relapsed or refractory, indolent or aggressive lymphoma. Annals of oncology : official journal of the European Society for Medical Oncology 2017; 28(9): 21692178.

30. Palomero T, Sulis ML, Cortina M, Real PJ, Barnes K, Ciofani M et al. Mutational loss of PTEN induces resistance to NOTCH1 inhibition in T-cell leukemia. Nature medicine 2007; 13(10): 1203-10.

31. Attar N, Kurdistani SK. Exploitation of EP300 and CREBBP Lysine Acetyltransferases by Cancer. Cold Spring Harbor perspectives in medicine 2017; 7(3).

32. Schulz WA, Lang A, Koch J, Greife A. The histone demethylase UTX/KDM6A in cancer: Progress and puzzles. International journal of cancer 2019; 145(3): 614-620.

33. Yang H, Jeffrey PD, Miller J, Kinnucan E, Sun Y, Thoma NH et al. BRCA2 function in DNA binding and recombination from a BRCA2-DSS1-ssDNA structure. Science (New York, N. Y.) 2002; 297(5588): 1837-48.

34. Holloman WK. Unraveling the mechanism of BRCA2 in homologous recombination. Nature structural \& molecular biology 2011; 18(7): 748-54.

35. Papadimitriou M, Mountzios G, Papadimitriou CA. The role of PARP inhibition in triple-negative breast cancer: Unraveling the wide spectrum of synthetic lethality. Cancer treatment reviews 2018; 67: 34-44.

36. Yin L, Liu Y, Peng Y, Peng Y, Yu X, Gao Y et al. PARP inhibitor veliparib and HDAC inhibitor SAHA synergistically co-target the UHRF1/BRCA1 DNA damage repair complex in prostate cancer cells. Journal of experimental \& clinical cancer research : CR 2018; 37(1): 153.

37. Pilarski R. The Role of BRCA Testing in Hereditary Pancreatic and Prostate Cancer Families. American Society of Clinical Oncology educational book. American Society of Clinical Oncology. Annual Meeting 2019; 39: 79-86.

38. Suárez C, Barnes L, Silver CE, Rodrigo JP, Shah JP, Triantafyllou A et al. Cervical lymph node metastasis in adenoid cystic carcinoma of oral cavity and oropharynx: A collective international review. Auris, nasus, larynx 2016; 43(5): 477-84.

39. Chen L, Yang L, Yao L, Kuang XY, Zuo WJ, Li S et al. Characterization of PIK3CA and PIK3R1 somatic mutations in Chinese breast cancer patients. Nature communications 2018; 9(1): 1357.

40. Kalev P, Simicek M, Vazquez I, Munck S, Chen L, Soin T et al. Loss of PPP2R2A inhibits homologous recombination DNA repair and predicts tumor sensitivity to PARP inhibition. Cancer research 2012; 72(24): 6414-24.

41. Szanto PA, Luna MA, Tortoledo ME, White RA. Histologic grading of adenoid cystic carcinoma of the salivary glands. Cancer 1984; 54(6): 1062-9.

42. Kazunori N, Keishi Y, Hiromichi S, Mina W, Hiroshi K, Nobukazu N et al. Aberrant methylation of GCNT2 is tightly related to lymph node metastasis of primary CRC. 2015; 35(3): 1411-21.

43. Han J, Zhang C, Gu T, Yang X, Hu L, Tian Z et al. Analysis of clinicopathological characteristics, MYB rearrangement and prognostic factors in salivary adenoid cystic carcinoma. Oncology letters 2019; 
17(3): 2915-2922.

44. Gerlinger M, Rowan AJ, Horswell S, Math M, Larkin J, Endesfelder D et al. Intratumor heterogeneity and branched evolution revealed by multiregion sequencing. The New England journal of medicine 2012; 366(10): 883-892.

45. Fusco N, Geyer FC, De Filippo MR, Martelotto LG, Ng CK, Piscuoglio S et al. Genetic events in the progression of adenoid cystic carcinoma of the breast to high-grade triple-negative breast cancer. Modern pathology : an official journal of the United States and Canadian Academy of Pathology, Inc 2016; 29(11): 1292-1305.

\section{Tables}

Due to technical limitations, table 1, 2 and 3 is only available as a download in the Supplemental Files section.

\section{Figures}

A

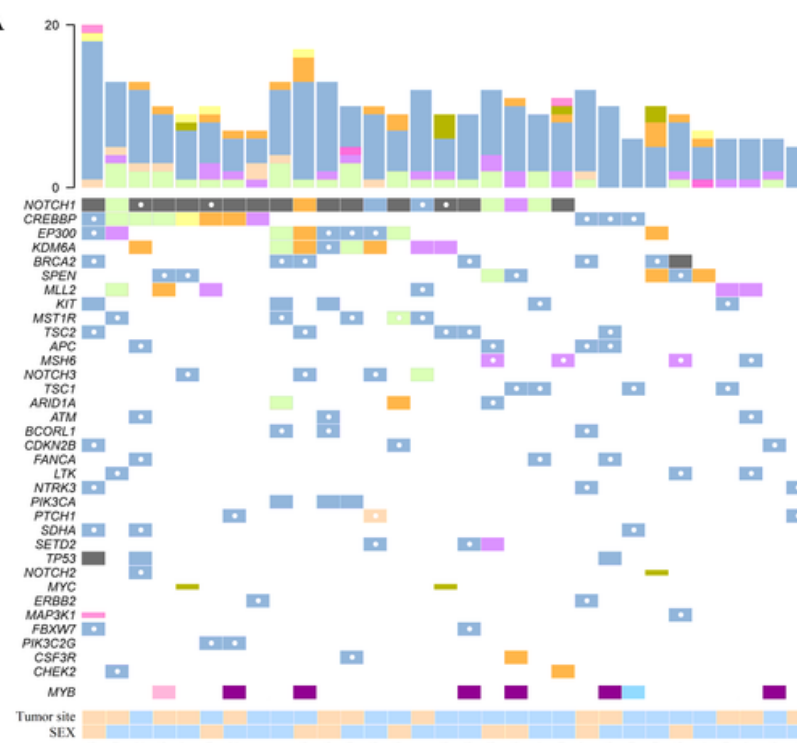

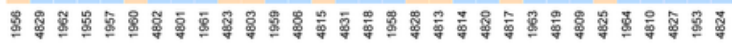

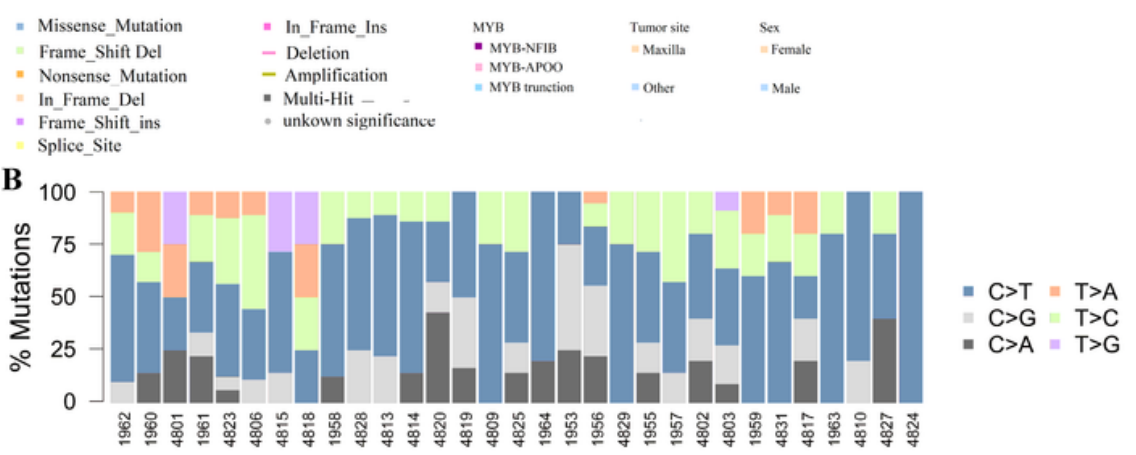

C
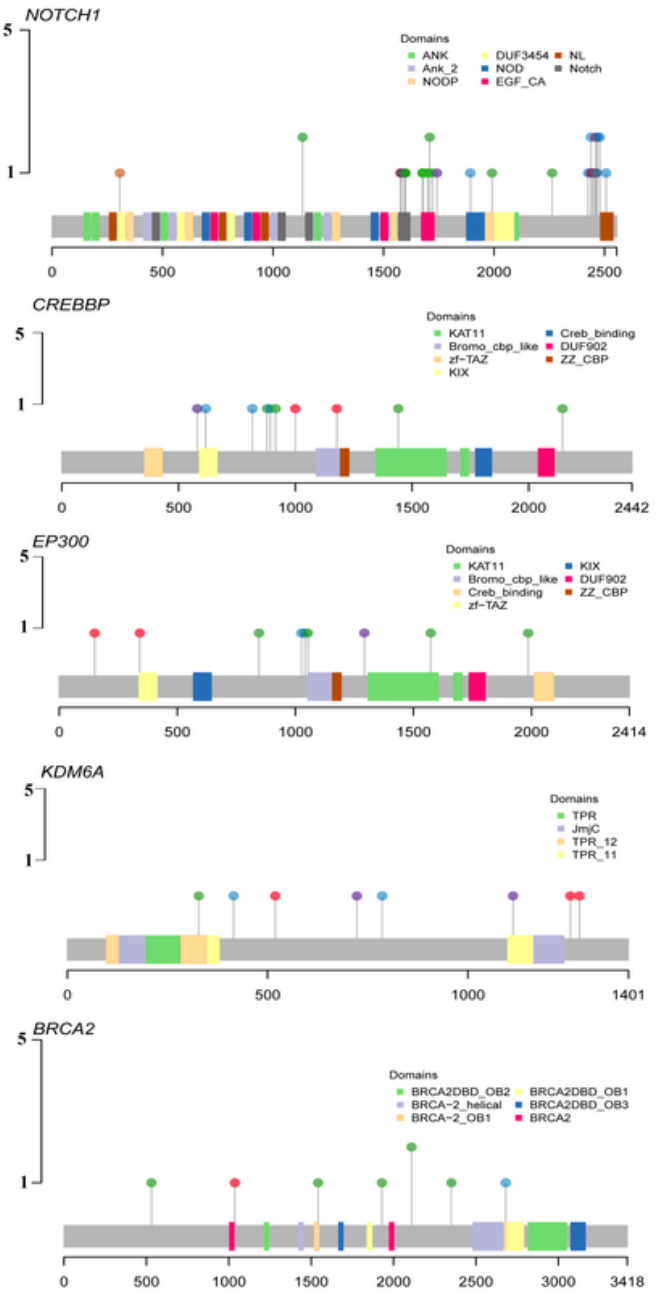

Figure 1 
The genetic profiles of solid type ACCs. (A) Oncoprints of recurrent mutations and copy number variants of 31 cases of solid type ACCs. (B) The mutation spectrum per sample across the 31 solid type ACC cases. (C) Lollipop plots of mutations in key genes in the solid type ACCs: for NOTCH1 in particular, 46 distinct alterations were observed, with 40 found in established hot-spot regions and considered activating mutations.

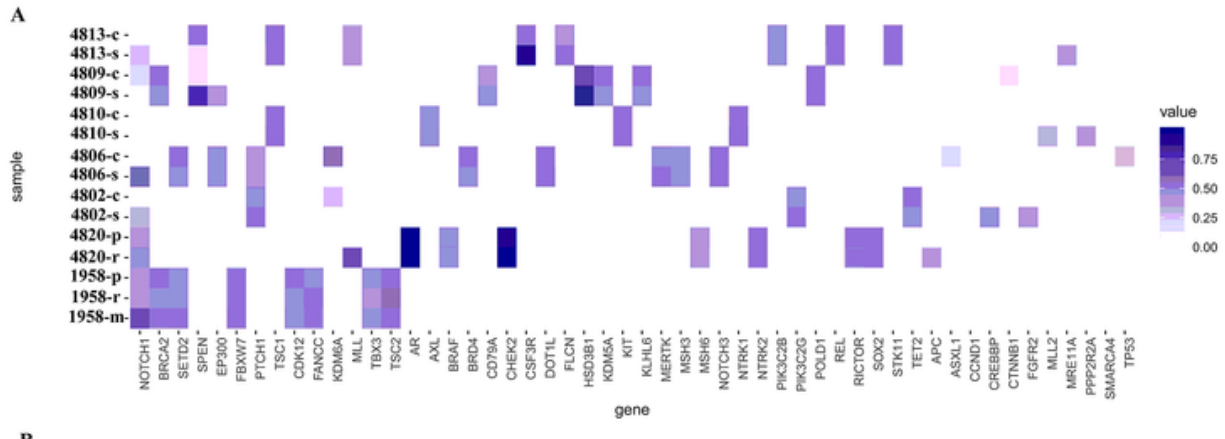

B
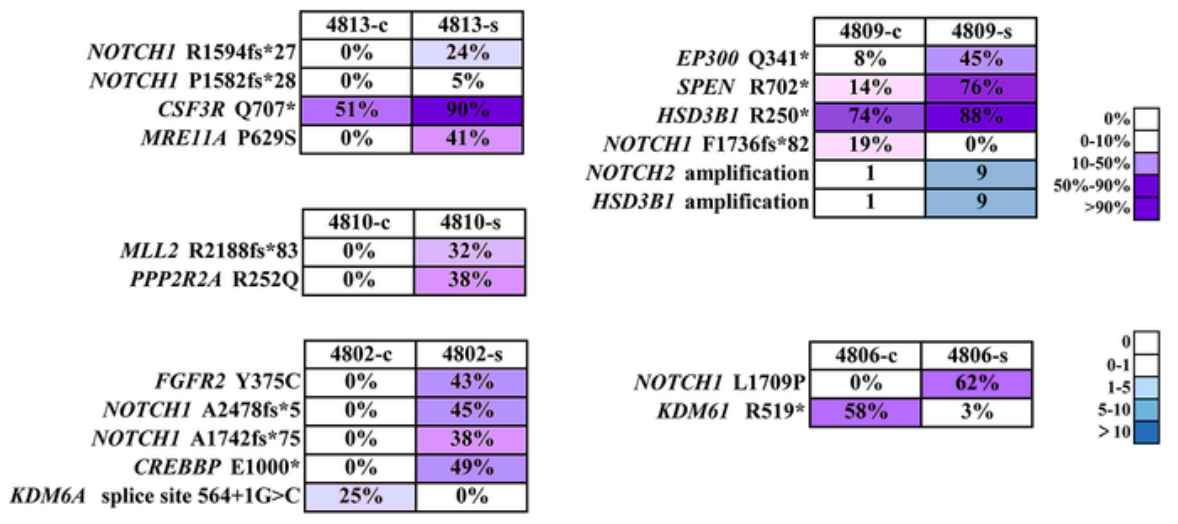

C
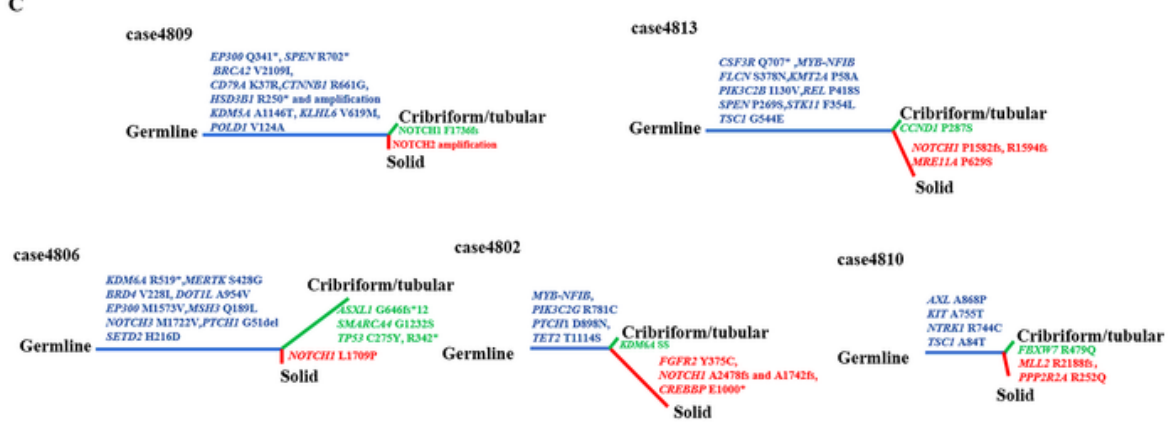

D

\begin{tabular}{r|c|c|}
\cline { 2 - 3 } APC G2597E & $4820-\mathrm{p}$ & $4820-\mathrm{r}$ \\
\cline { 2 - 3 } KMT2A R34L & $0 \%$ & $38 \%$ \\
\cline { 2 - 3 } HSD3B1 amplification & $0 \%$ & $64 \%$ \\
\cline { 2 - 3 } NOTCH2 amplification & 1 & 6 \\
\cline { 2 - 3 } & 1 & 6 \\
\hline
\end{tabular}

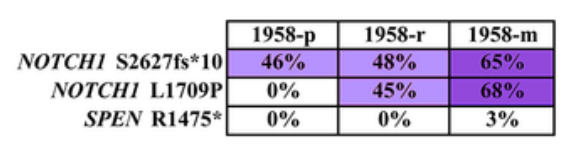

E
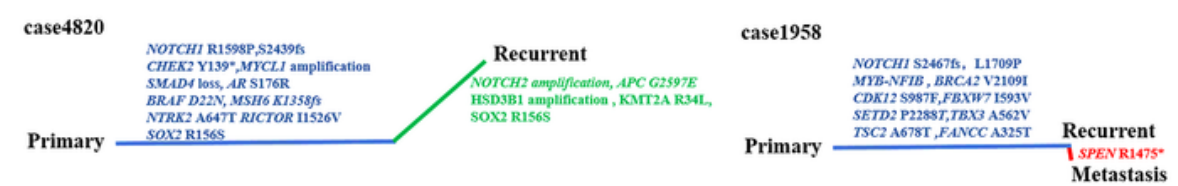

Figure 2 
The genetic alterations with the solid growth pattern component versus the cribriform/tubular component and the primary tumor, the local relapse versus the lung metastases. (A) Heatmap of cancer cell fractions of somatic mutations identified in the solid growth pattern component versus the cribriform/tubular component and the primary tumor, the local relapse versus the lung metastases. (B) Diagram highlighting the key genetic alterations identified in the cribriform/tubular component and the solid component in 5 ACC cases and in the primary tumor, the local relapse and the lung metastases in other 2 solid type ACC cases. Cancer cell fractions are shown, which are the estimated percentage of cancer cells harboring a given somatic mutation, and are color-coded according to the legend. In the progression from the cribriform/tubular to the solid type ACC, the subclonal mutation of NOTCH1, MRELLA, MLL2, PPP2R2A and FGFR2 and the gain of copy number in NOTCH2 were acquired.(C) Phylogenetic tree showing the evolution of the cribriform/tubular adenoid cystic carcinoma and the solid components, where the colored branches represent each of the subclones identified. The length of the branches is representative of the number of mutations and copy number alterations that distinguishes a given clone from its ancestral clone; somatic genetic alterations that define a given subclone are illustrated along the branches45. Ubiquitous mutations (blue): The mutations can be found in both components of high-grade ACC. Cribriform/tubular private mutations (green): The mutations can only be found in the cribriform/tubular components. Solid private mutations (red): The mutations can only be found in only solid components. (D) Diagram highlighting the key genetic alterations identified in the primary tumor, the local relapse and the lung metastases of $2 \mathrm{ACC}$ cases. In the development in the local relapse, the subclonal mutation of APC and KMT2A, and gains in the chromosome 1 (NOTCH2 and HSD3B1) were obtained. In the development of lung metastases, the genetic profiles of the primary tumors and lung metastatic nodes are highly similar, but with the NOTCH1 mutation enriched. (E) The phylogenetic tree of somatic mutations for 5 tumor tissues from 2 ACCs. Recurrent private mutations (green): The mutations can only be found in the recurrent samples. Metastasis private mutations (red): The mutations can only be found in the metastatic tissues. The immunostaining of NCID in the solid component with NOTCH1 mutation was much stronger than the cribriform/tubular component with no NOTCH1 mutation. 


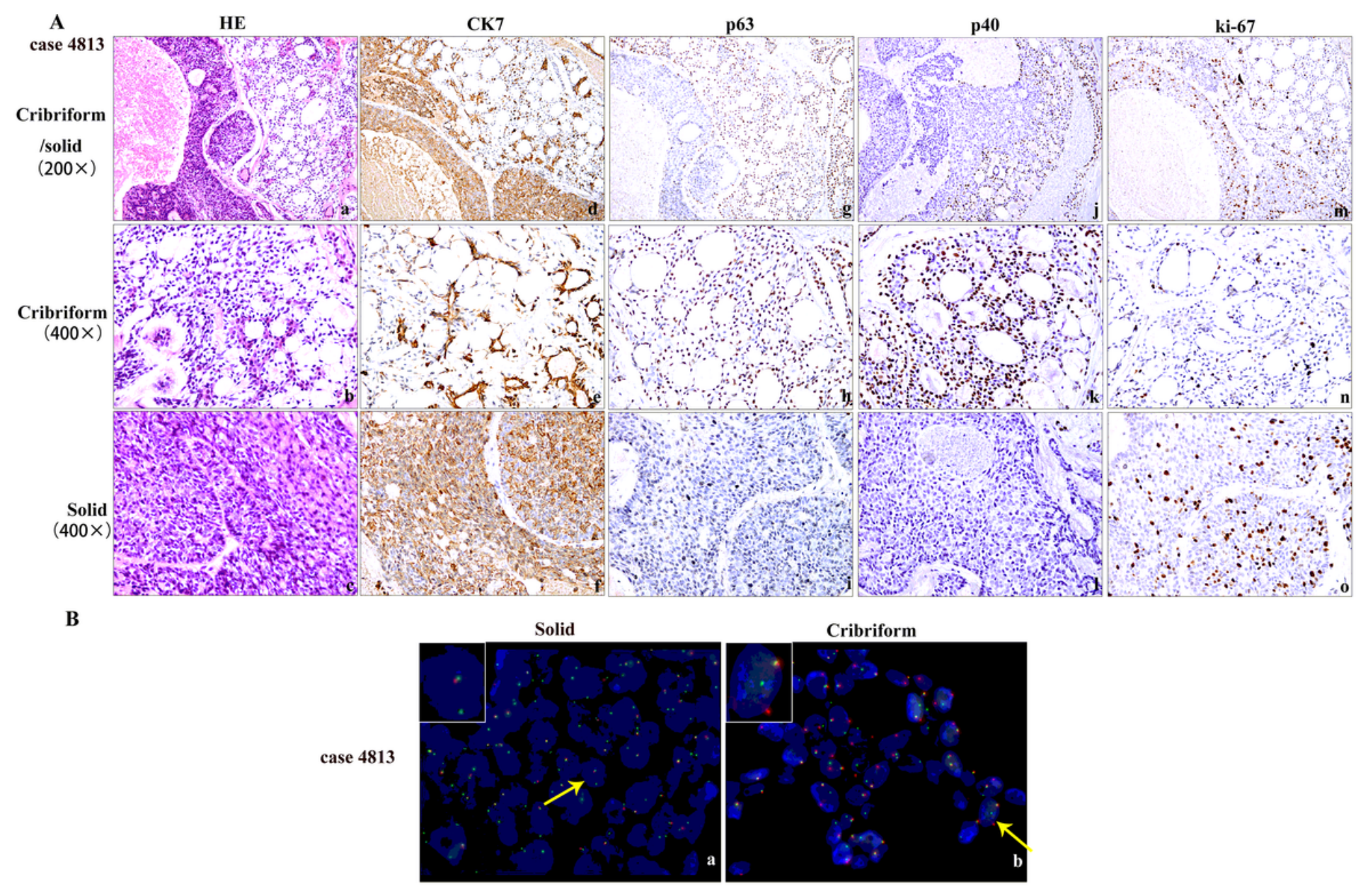

\section{Figure 3}

Histopathologic and immunohistochemical characteristics of solid type ACC. (A) Morphology (a, g, m) (hematoxylin \& eosin stains) showing the solid type ACC 4813 arranged in two distinct histologic patterns $(a, 200 x)$ : the cribriform growth pattern $(m, 400 x)$ and a solid growth pattern $(g, 400 x)$. P63 immunostaining $(\mathrm{g}, \mathrm{h}, \mathrm{i})$ and $\mathrm{p} 40(\mathrm{j}, \mathrm{k}, \mathrm{l})$ is positive in the myoepithelial cell in the cribriform/tubular component and negative in the solid component while CK7 immunostaining $(d, e, f)$ is positive in the gland epithelium cell and diffusely positive in the solid component. Ki-67 immunostaining $(m, n, 0)$ was nuclear $10 \%$ positive in the solid component and $₫ 5 \%$ in the cribriform/tubular component. (B) In the same case of ACC 4813 arranged in different patterns, FISH analysis showed that the typical patterns with an intact signal (fused orange/green signals) and a split signal (separated orange and green signals) was shown in the cribriform components (b), while the atypical pattern with one fusion signal and one green signal was present in the solid components (a). 
A

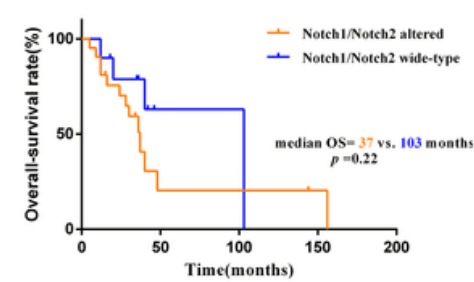

B

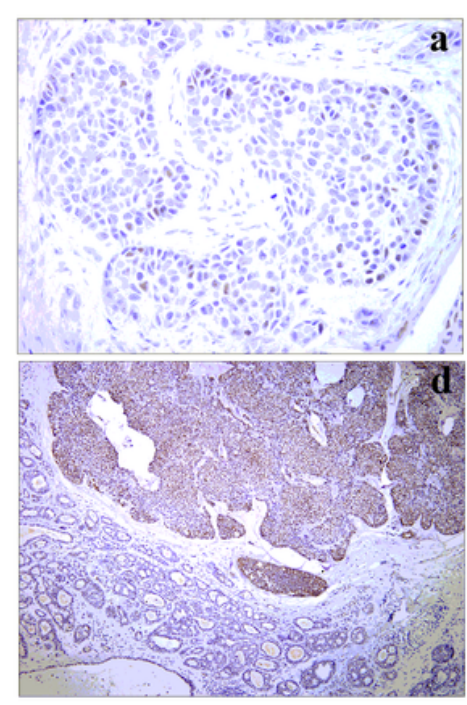

C

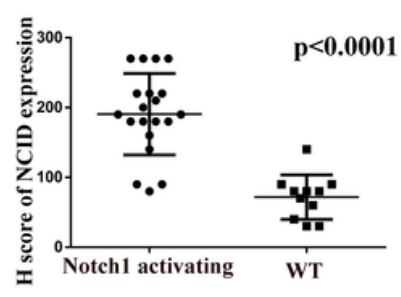

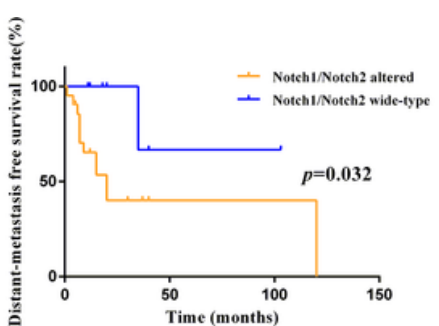
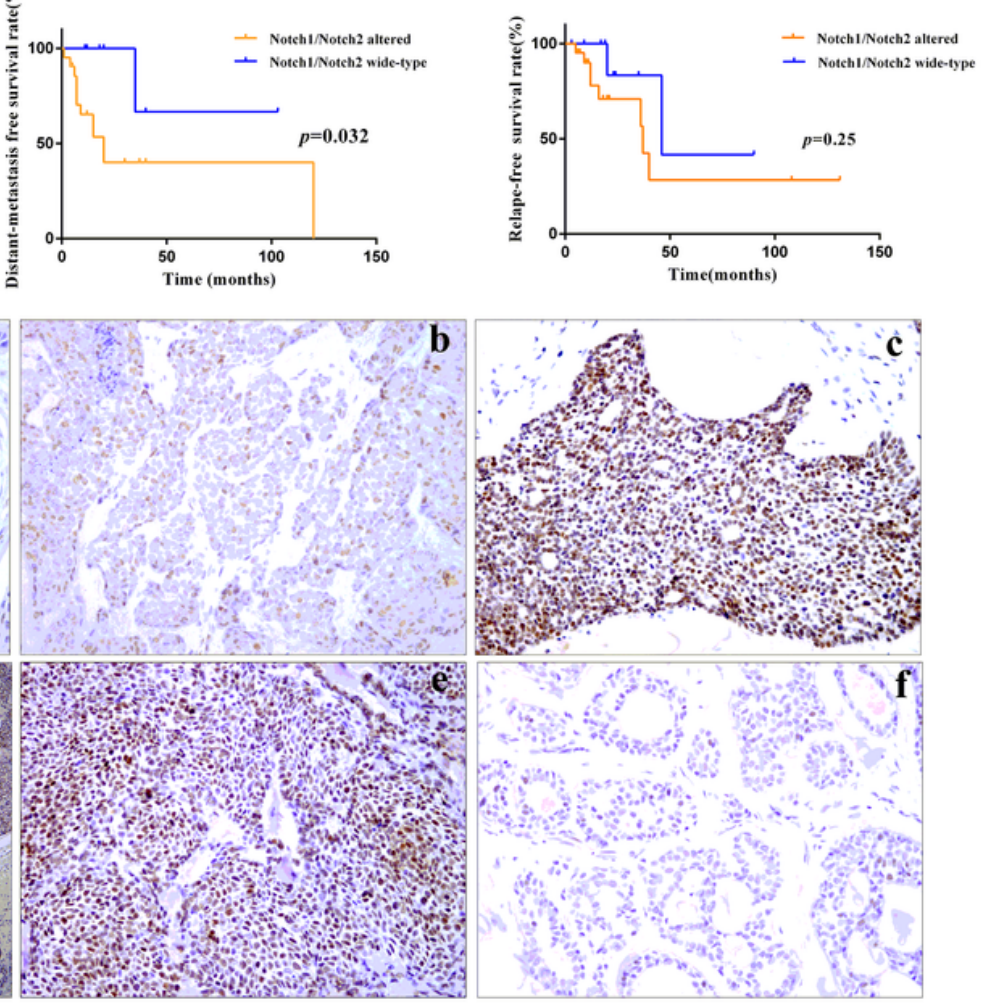

D

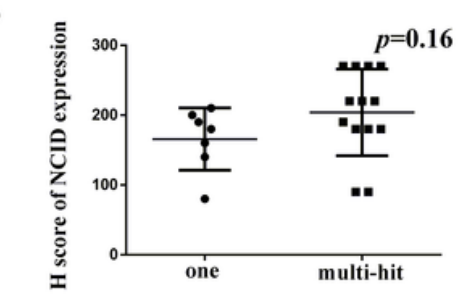

\section{Figure 4}

A high NCID expression level is associated with poorer associated with poorer distant metastasis-free survival. (A)The Kaplan-Meier analysis was used to compare the overall survival, distant metastasis-free survival and recurrence-free survival between the cases with NOTCH1 mutation and without NOTCH1 mutation in the 31 cases of solid type ACC. (B) Representative images of the IHC staining of NICD in paraffin-embedded tissues of solid type ACCs (a negative, b weak, c strong) and in the different components of case4812( $d$ mixed region, e solid, $f$ cribriform). (C) A statistically significant association between NOTCH1 mutations and NICD positivity was identified. (D) No statistically significant difference of NICD1 expression between tumors with one and multi-hit NOTCH1 mutations. 


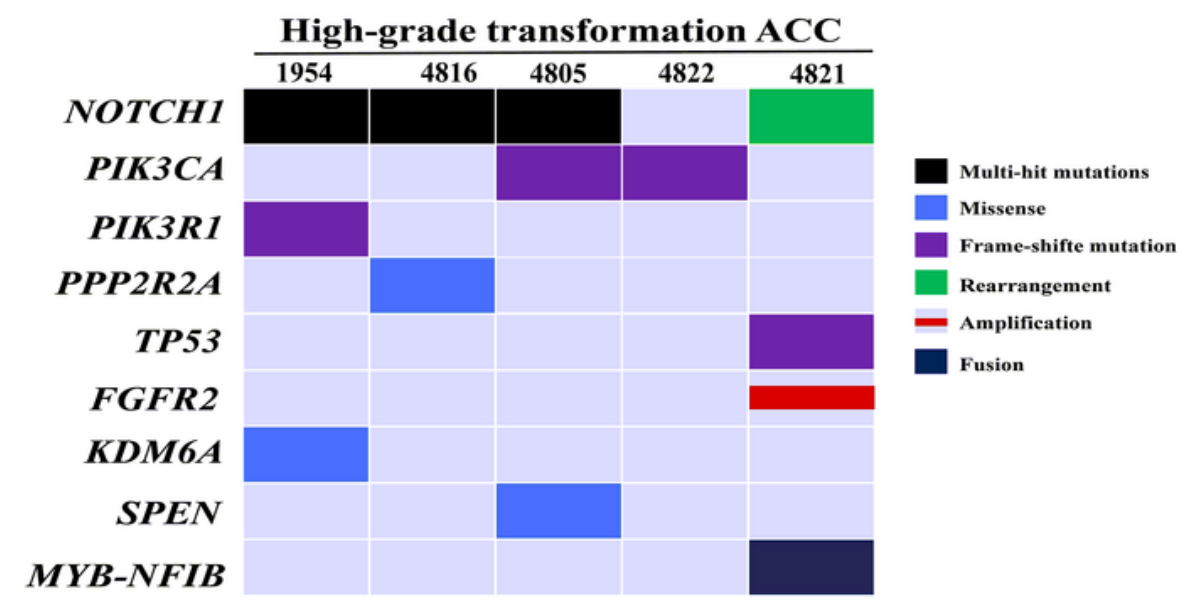

B

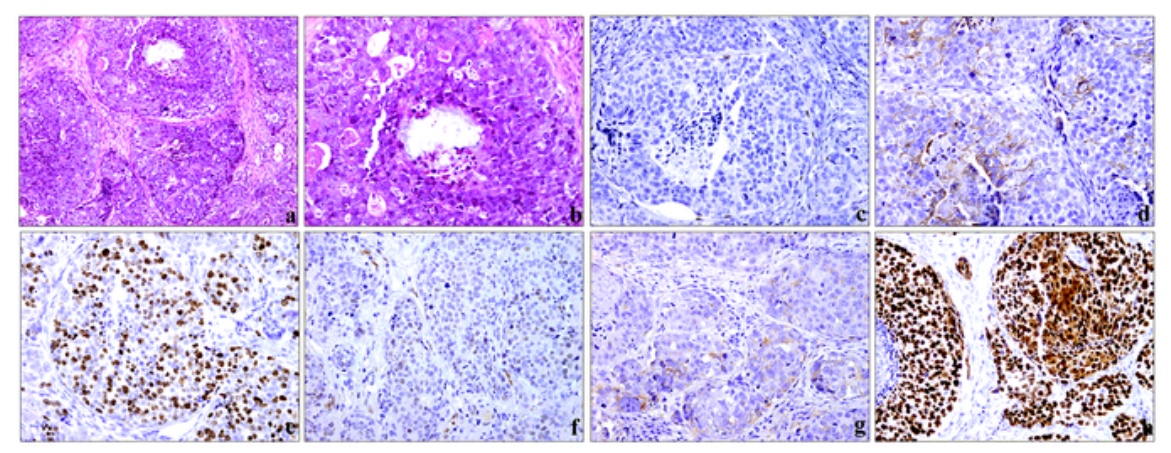

C

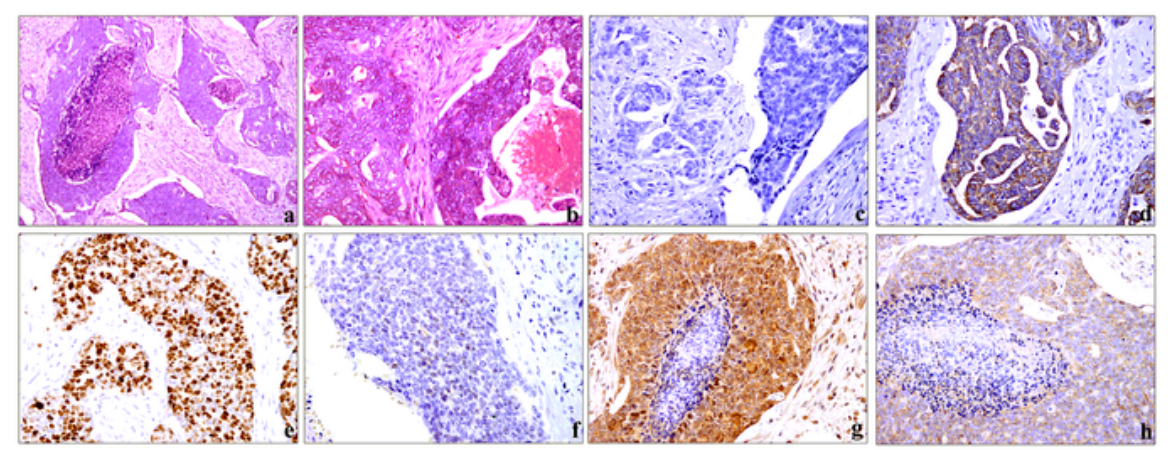

\section{Figure 5}

The critical genetic alterations of ACC-HGT. (A) The waterfall plot reveals common mutations in NOTCH1, genes associated with PI3K pathway, p53 and FGFR2 amplification for the ACC with HGT. (B) The case of ACC-HGT 4821 with a frame-shift mutation in p53. Morphology (hematoxylin \& eosin stains) showing the ACC with HGT in original magnification of $200 \times(a)$ and $400 \times(b)$. The transformed ACC consists of sheets of atypical cells and loss of architecture usually seen in a conventional ACC with prominent nucleoli and 
necrosis. p63 immunostaining(c) was negative and CK7 immunostaining(d) was weakly positive in the high-transformation grade area. The Ki-67 immunostaining(e) was with more than $50 \%$ positivity, indicating a high proliferation ability. The NCID(f) and p-AKTT308(g) immunostaining was negative. A strong and diffuse expression of P53 was observed(h). (C) The case of ACC-HGT 4822. Morphology (hematoxylin \& eosin stains) showing the ACC with HGT in original magnification of $200 \times(a)$ and $400 \times(b)$. The transformed component shows a micropapillary growth pattern in a desmoplastic stroma with more nuclear enlargement, pleomorphism, and prominent nucleoli. p63 immunostaining(c) was negative and CK7 immunostaining(d) was moderately positive. The Ki-67 immunostaining(e) was more than $50 \%$ positive, indicating a high proliferation ability. The NCID(f) immunostaining was negative, while the PI3 Kinase p110a and p-AKTT308 immunostaining were strongly positive. 


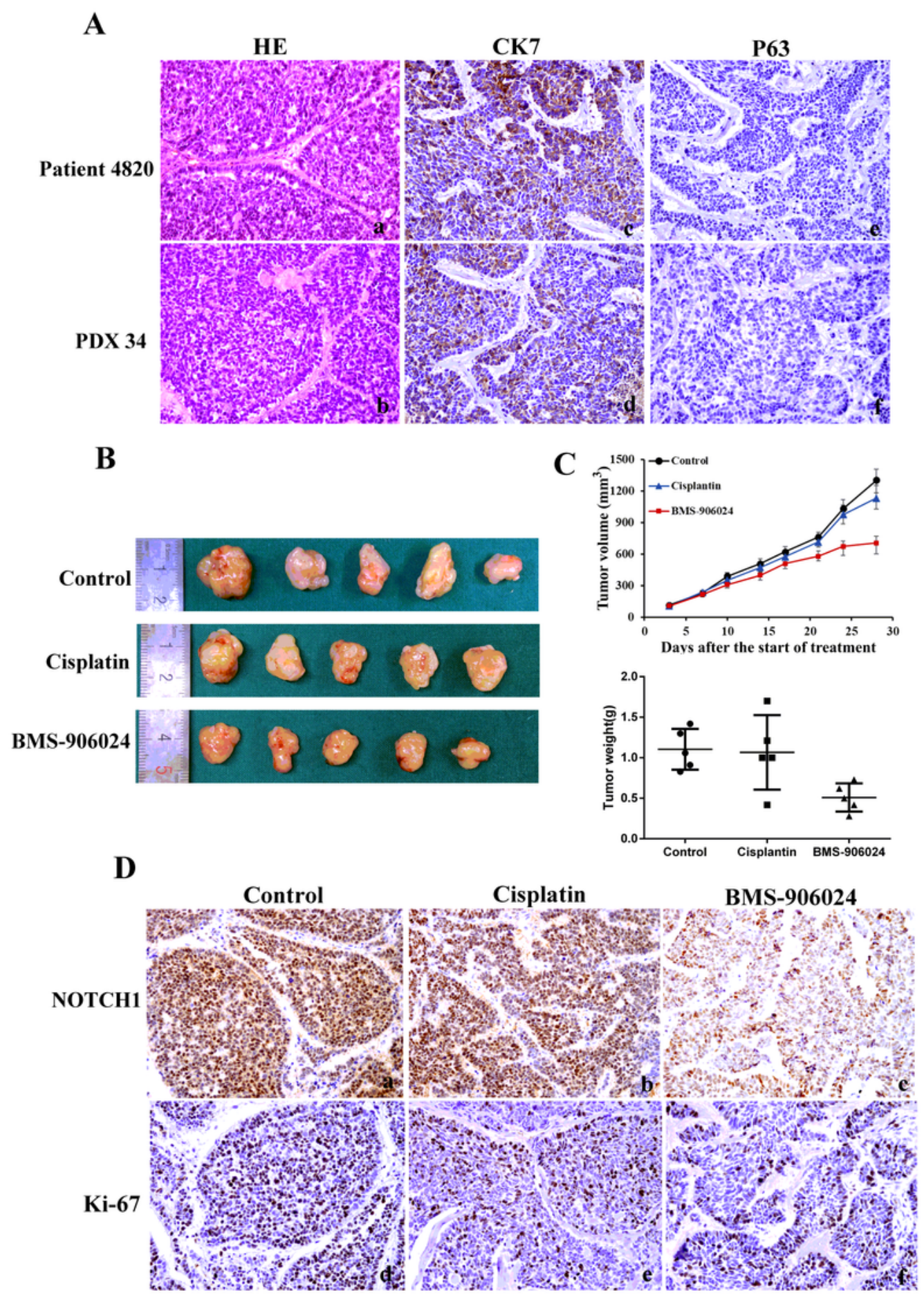

\section{Figure 6}

The in vivo evaluation of BMS-906024 in high grade ACC PDXs. (A) PDX showed histological consistency to ACC 4820. The xenograft tumor exhibited solid type $(a, b)$ and diffusely positive for CK7(c,d) and negative for p63(e,f), similar to ACC 4820. (B, C) BMS-906024(TGI=48\%) reduced the volume and weight of xenograft tumors and exhibited a better tumor growth inhibitory effect than cisplatin (TGI $=12 \%$ ). (D) IHC staining of NCID in the PDX tumors after 28 days of treatment. $(a, b, c)$ Treatment of PDX 34 with 
BMS-906024 inhibited NCID expression much better than cisplatin and the control. (d,e,f) Cell proliferation, which assessed by Ki67 staining, was reduced to varying degrees in the cisplatin and BMS906024 groups.

\section{Supplementary Files}

This is a list of supplementary files associated with this preprint. Click to download.

- genealterations1.xlsx

- genealterations2.xlsx

- supplementarydata.docx

- Table1.pdf

- Table2.pdf

- Table3.pdf 